Mitigation of Laser Damage Growth in Fused Silica with a Galvanometer Scanned CO2 Laser

I. L. Bass, G. M. Guss, R. P. Hackel

November 1, 2005

Boulder Damage Symposium XXXVII

Boulder, CO, United States

September 19, 2005 through September 21, 2005 
This document was prepared as an account of work sponsored by an agency of the United States Government. Neither the United States Government nor the University of California nor any of their employees, makes any warranty, express or implied, or assumes any legal liability or responsibility for the accuracy, completeness, or usefulness of any information, apparatus, product, or process disclosed, or represents that its use would not infringe privately owned rights. Reference herein to any specific commercial product, process, or service by trade name, trademark, manufacturer, or otherwise, does not necessarily constitute or imply its endorsement, recommendation, or favoring by the United States Government or the University of California. The views and opinions of authors expressed herein do not necessarily state or reflect those of the United States Government or the University of California, and shall not be used for advertising or product endorsement purposes.

This work was performed under the auspices of the U.S. Department of Energy by University of California, Lawrence Livermore National Laboratory under Contract W-7405-Eng-48. 


\title{
Mitigation of Laser Damage Growth in Fused Silica with a Galvanometer Scanned $\mathrm{CO}_{2}$ Laser
}

\author{
Isaac L. Bass*, Gabriel M. Guss, Richard P. Hackel \\ Lawrence Livermore National Laboratory \\ 7000 East Avenue, Mailstop L-592 \\ Livermore, CA 94550
}

\begin{abstract}
At the National Ignition Facility (NIF) at the Lawrence Livermore National Laboratory (LLNL), mitigation of laser surface damage growth on fused silica using single and multiple $\mathrm{CO}_{2}$ laser pulses has been consistently successful for damage sites whose lateral dimensions are less than $100 \mu \mathrm{m}$, but has not been for larger sites. Cracks would often radiate outward from the damage when a $\mathrm{CO}_{2}$ pulse was applied to the larger sites. An investigation was conducted to mitigate large surface damage sites using galvanometer scanning of a tightly focused $\mathrm{CO}_{2}$ laser spot over an area encompassing the laser damage. It was thought that by initially scanning the $\mathrm{CO}_{2}$ spot outside the damage site, radiating crack propagation would be inhibited. Scan patterns were typically inward moving spirals starting at radii somewhat larger than that of the damage site. The duration of the mitigation spiral pattern was $\sim 110 \mathrm{~ms}$ during which a total of $\sim 1.3 \mathrm{~J}$ of energy was delivered to the sample. The $\mathrm{CO}_{2}$ laser spot had a $1 / \mathrm{e}^{2}$-diameter of $\sim 200 \mu \mathrm{m}$. Thus, there was general heating of a large area around the damage site while rapid evaporation occurred locally at the laser spot position in the spiral. A 30 to $40 \mu \mathrm{m}$ deep crater was typically generated by this spiral with a diameter of $\sim 600 \mu \mathrm{m}$. The spiral would be repeated until there was no evidence of the original damage in microscope images. Using this technique, damage sites as large as $300 \mu \mathrm{m}$ in size did not display new damage after mitigation when exposed to fluences exceeding $22 \mathrm{~J} / \mathrm{cm}^{2}$ at $355 \mathrm{~nm}, 7.5 \mathrm{~ns}$. It was found necessary to use a vacuum nozzle during the mitigation process to reduce the amount of re-deposited fused silica. In addition, curing spiral patterns at lower laser powers were used to presumably "re-melt" any re-deposited fused silica. A compact, shearing interferometer microscope was developed to permit in situ measurement of the depth of mitigation sites.
\end{abstract}

Keywords: Mitigation, $\mathrm{CO}_{2}$, fused silica, laser damage, galvanometer, scanning

\section{INTRODUCTION}

The National Ignition Facility (NIF) at the Lawrence Livermore National Laboratory (LLNL) is designed to ignite a deuterium-tritium target with $\leq 1.8 \mathrm{MJ}$ of $351 \mathrm{~nm}$, UV laser light. This UV light is produced by tripling the amplified $1053 \mathrm{~nm}$ beam just before the beam is focused onto the target. Surface damage to the final fused silica optics limit the maximum fluence at which the NIF can operate. Some damage to these optics is expected at the fluences planned for NIF operation. Economic operation of the NIF will depend on mitigating (preventing) damage growth on the large, high-cost, final optics before it renders them useless.

It has been demonstrated that local evaporation and melting of fused silica by a $10.6 \mu \mathrm{m}, \mathrm{CO}_{2}$ laser pulse focused onto the damage site will prevent further growth ${ }^{1,2}$ of the damage. This method was proven successful provided that the initial damage site was less than $\sim 100 \mu \mathrm{m}$ in its transverse dimensions (i.e., average diameter). However, this was not the case for larger damage sites. Cracks would often propagate

\footnotetext{
* Correspondence: Email: bass1@1lnl.gov, Mailstop: L-592, Telephone: 925-423-8389, Fax: 925-422-5099
} 
away from the damage when the $\mathrm{CO}_{2}$ beam was applied or when the site was subsequently exposed to $351 \mathrm{~nm}$ pulses at representative NIF fluences. It was thought that the stresses created by the rapid heating and cooling of the fused silica led to this crack propagation.

The ability to mitigate growth of larger damage sites is important to reduce the recycling frequency of the final optics for mitigation, and because some sites will be larger than $100 \mu \mathrm{m}$ when first created or detected. A strategy was proposed to inhibit lateral crack propagation by first melting and evaporating the fused silica around the perimeter of the damage site. This was accomplished by scanning the $\mathrm{CO}_{2}$ laser spot with a fast, galvanometer beam scanner. After several passes around the perimeter, the beam was spiraled inward to evaporate the material within the site. This technique proved successful in mitigating damage growth in sites as large as $300 \mu \mathrm{m}$ when exposed to $7.5 \mathrm{~ns}, 355 \mathrm{~nm}$ laser pulses to fluences of $22 \mathrm{~J} / \mathrm{cm}^{2}$ (equivalent to the highest fluences expected in the NIF $351 \mathrm{~nm}$ beams).

The scanning technique offers several benefits beyond preventing crack propagation. Scan patterns can be matched to the shape of the damage site. The scan rate and laser power can be adjusted dynamically to control the shape of the mitigation crater. It was also observed that re-condensed fused silica debris on the surface was prone to damage. The scanning technique at lower power was used to prevent this damage by remelting, or curing the debris.

We report on the methods and results of using galvanometer scanning to mitigate growth of surface damage sites in fused silica as large as $300 \mu \mathrm{m}$.

\section{EXPERIMENTAL ARRANGEMENT of LASER SCANNER}

The $\mathrm{TEM}_{00}$ output beam of a Coherent, GEM-30A, air-cooled $\mathrm{CO}_{2}$ laser was allowed to expand to a diameter of $\sim 25 \mathrm{~mm}$ at a 10 " focal-length, 2"-diameter, positive ZnSe lens. As shown in Figs. 1 and 2, the beam passed through a Nutfield, QS30, open frame, XY scanning head equipped with $30 \mathrm{~mm}$ aperture mirrors coated for $10.6 \mu \mathrm{m}$. The fused silica sample surface was located at the focal point of the beam where the $1 / \mathrm{e}^{2}-$ beam diameter was $\sim 200 \mu \mathrm{m}$. A polarization rotator and thin-film-polarizer upstream were used to control the laser power to the sample. The laser was pulsed at $5 \mathrm{kHz}$ with the pulse length dynamically variable from $\sim 10 \mu$ s to $\mathrm{CW}$.

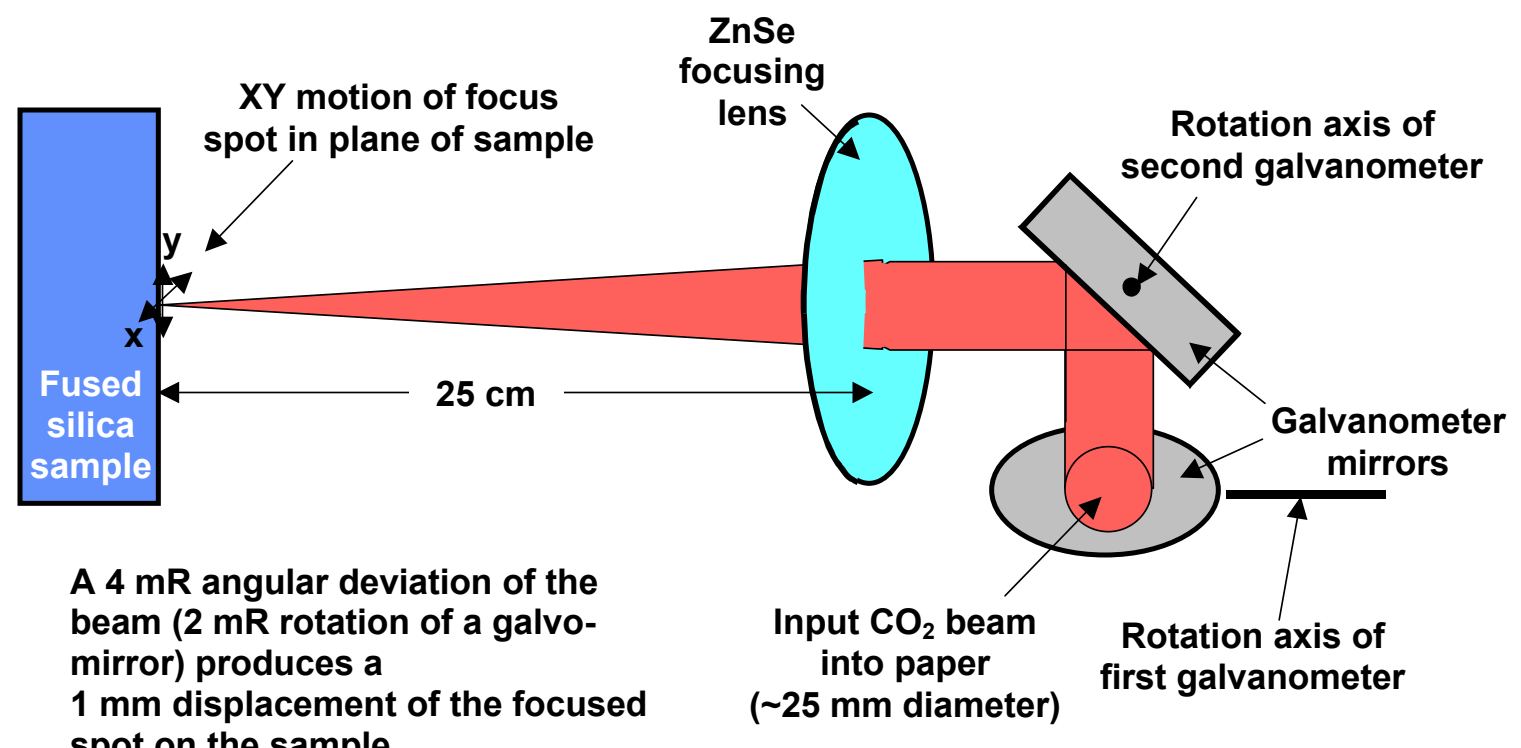


Fig. 1 Schematic diagram of the 2-axis, galvanometer for scanning the focused $\mathrm{CO}_{2}$ laser beam in patterns at the location of surface damage sites in fused silica.

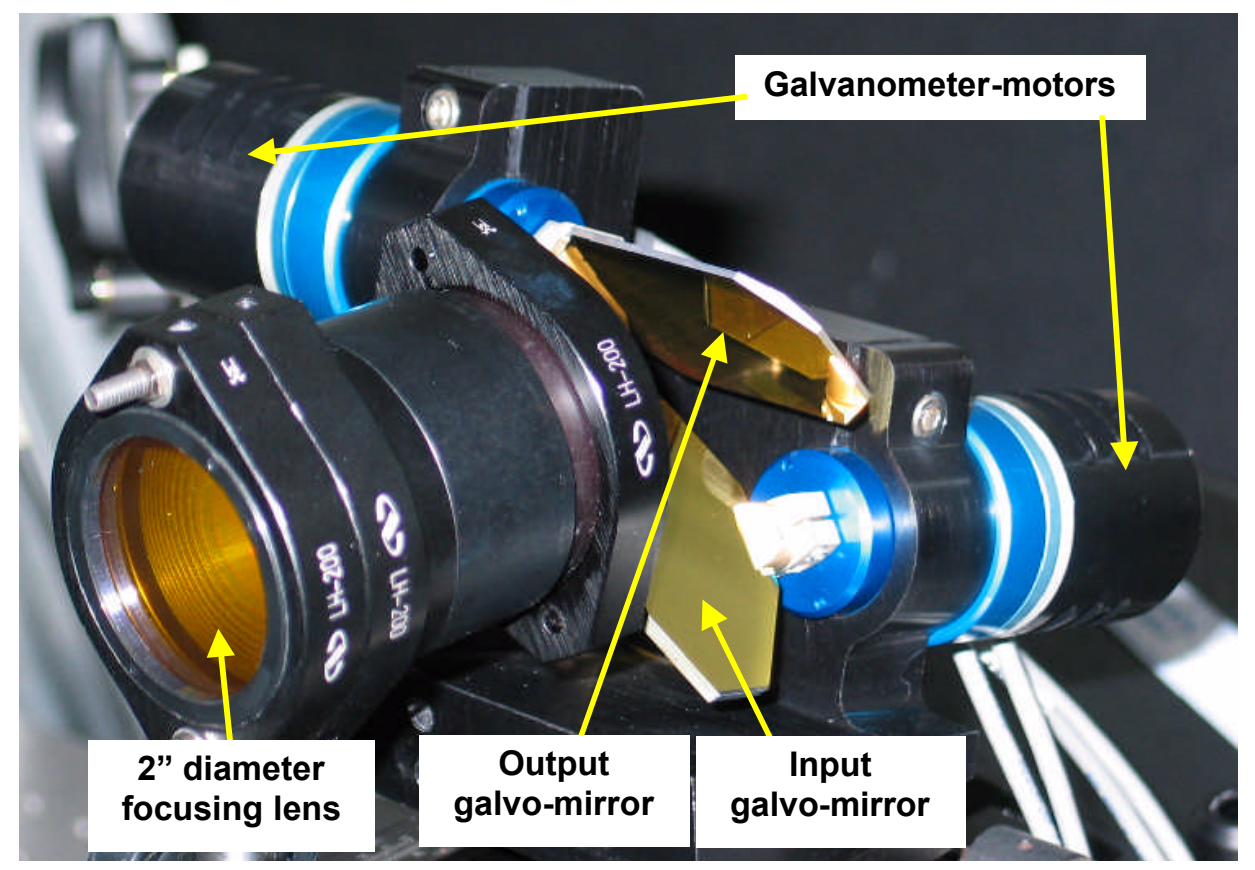

Fig. 2 Photograph of the XY galvanometer scanner and focusing lens.

\section{REDUCTION and CURING of FUSED SILICA DEBRIS}

The amount of fused silica evaporated in order to mitigate a damage site several hundred microns in diameter and $100 \mu \mathrm{m}$ or more deep leads to a significant amount of fused silica re-deposited or re-condensed as debris on the surface. This is evident in Fig. 3 where the fused silica vapor was intentionally blown to the side by a jet of air. It was observed that this debris is prone to laser damage. In order to reduce the amount of debris, a nozzle was designed to vacuum the fused silica vapor away from the surface as shown in Fig. 4. 


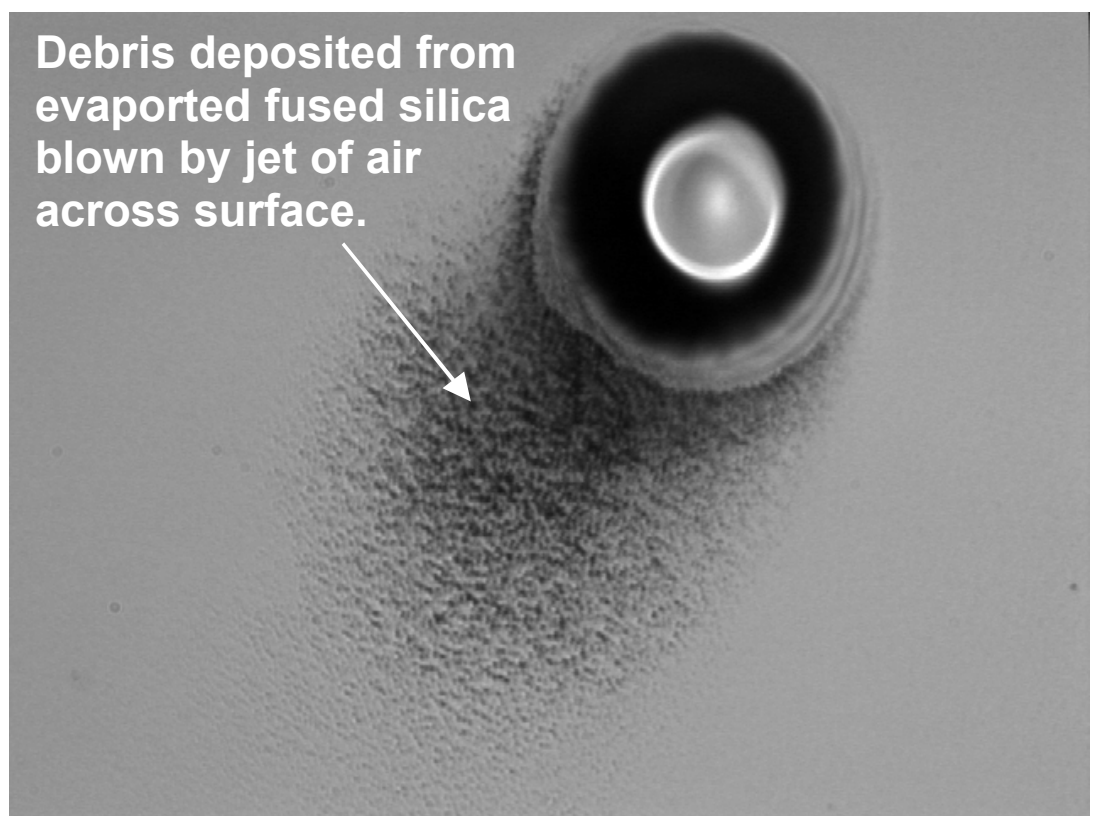

Fig. 3 Re-deposited or re-condensed fused silica debris is clearly evident.

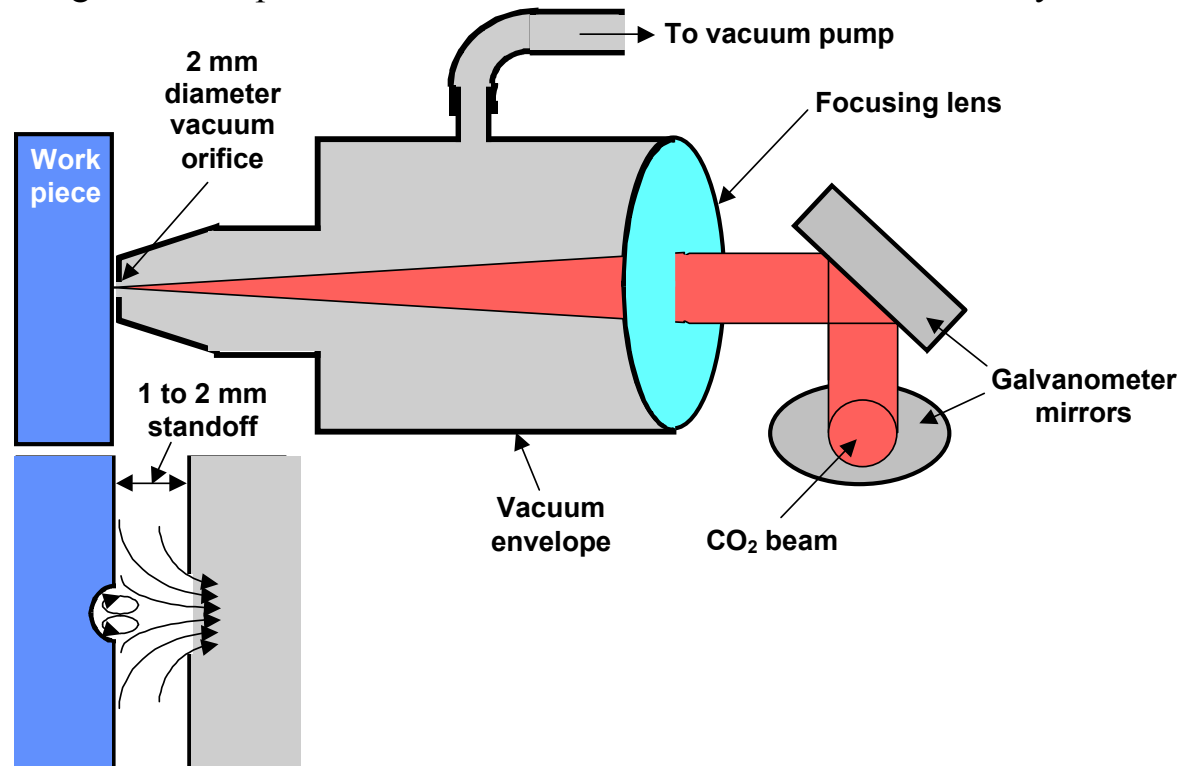




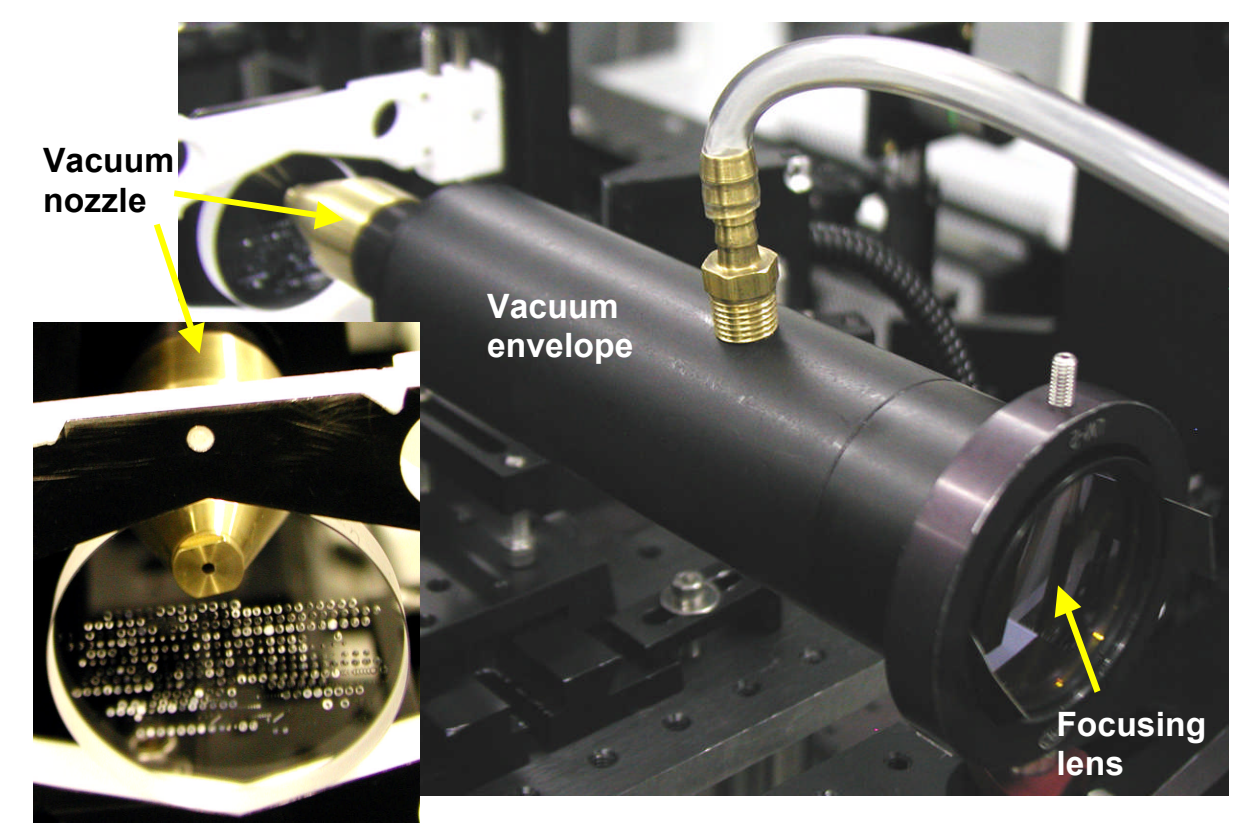

Fig. 4 Schematic diagram and photograph of the vacuum nozzle assembly designed to reduce the amount of evaporated fused silica that re-deposits or re-condenses as debris on the surface during the mitigation process.

It was also observed that the tendency of the fused silica debris to damage could be reduced by "re-melting" it with several, lower power "curing" spiral scans following the initial high power evaporation, or "cutting" spiral scan. The curing spiral powers were set near or below the evaporation threshold so that they generated a negligible amount of additional debris. The effect of vacuum and curing on the amount and visibility of the debris can be seen in Fig. 5 where two different techniques of oblique illumination were used to suppress specular reflections and highlight the debris in scattered light. 


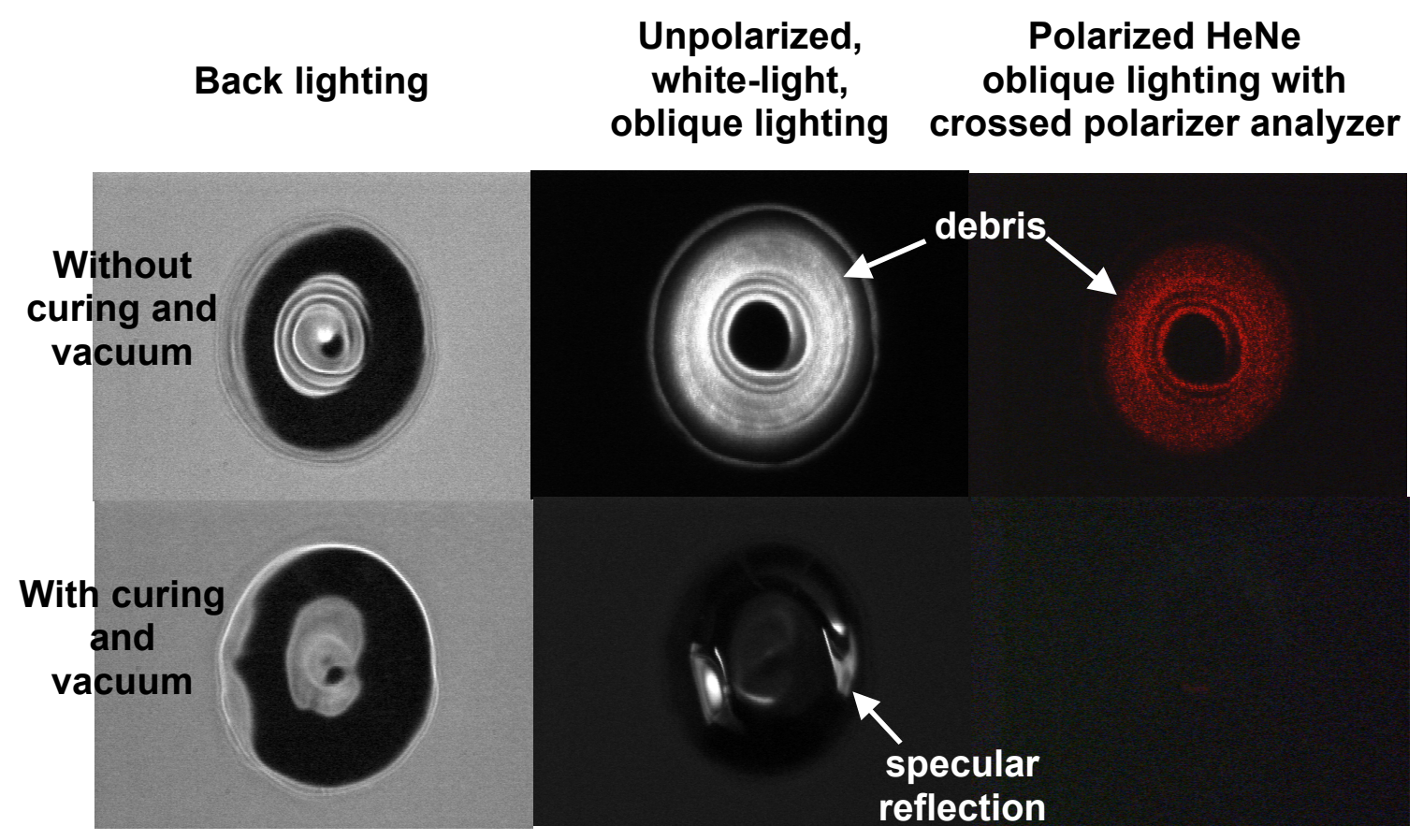

Fig. 4 Images of a mitigation site generated by evaporation spiral scans without vacuum and subsequent curing scans (top row), and with vacuum and subsequent curing scans (bottom row). The effectiveness of vacuum and curing on reducing the amount of residual debris can be seen clearly in the two sets of images on the right where oblique lighting suppresses specular reflections and highlights the debris in scattered light.

\section{MITIGATION and CURING SPIRAL PROTOCOLS}

Spiral mitigation protocols were developed to remove fused silica to depths of the order of 50 microns while being able to resist damage to representative NIF fluences. Fig. 5 illustrates one such protocol (or recipe) schematically that includes three subsequent curing spirals. Laser power, laser duty factor (pulse length), scan velocity, and spiral pitch (radial rate) are adjusted to optimize the shape, depth, and damage resistance of the mitigation crater. A microscope shearing interferometer was used to measure depths on-line and facilitated optimization. This particular protocol produced craters $\sim 750 \mu \mathrm{m}$ in diameter and $60 \mu \mathrm{m}$ deep (see Fig. 6). In order to mitigate deeper, the protocol was repeated multiple times. The radial scale of the pattern was increased by $\sim 3 \%$ on each pass. The achievable depth eventually saturated near $200 \mu \mathrm{m}$. We believe this was due to increasing reflection and outward refraction of the incident beam on the walls of the crater as they became steeper with increasing depth. 


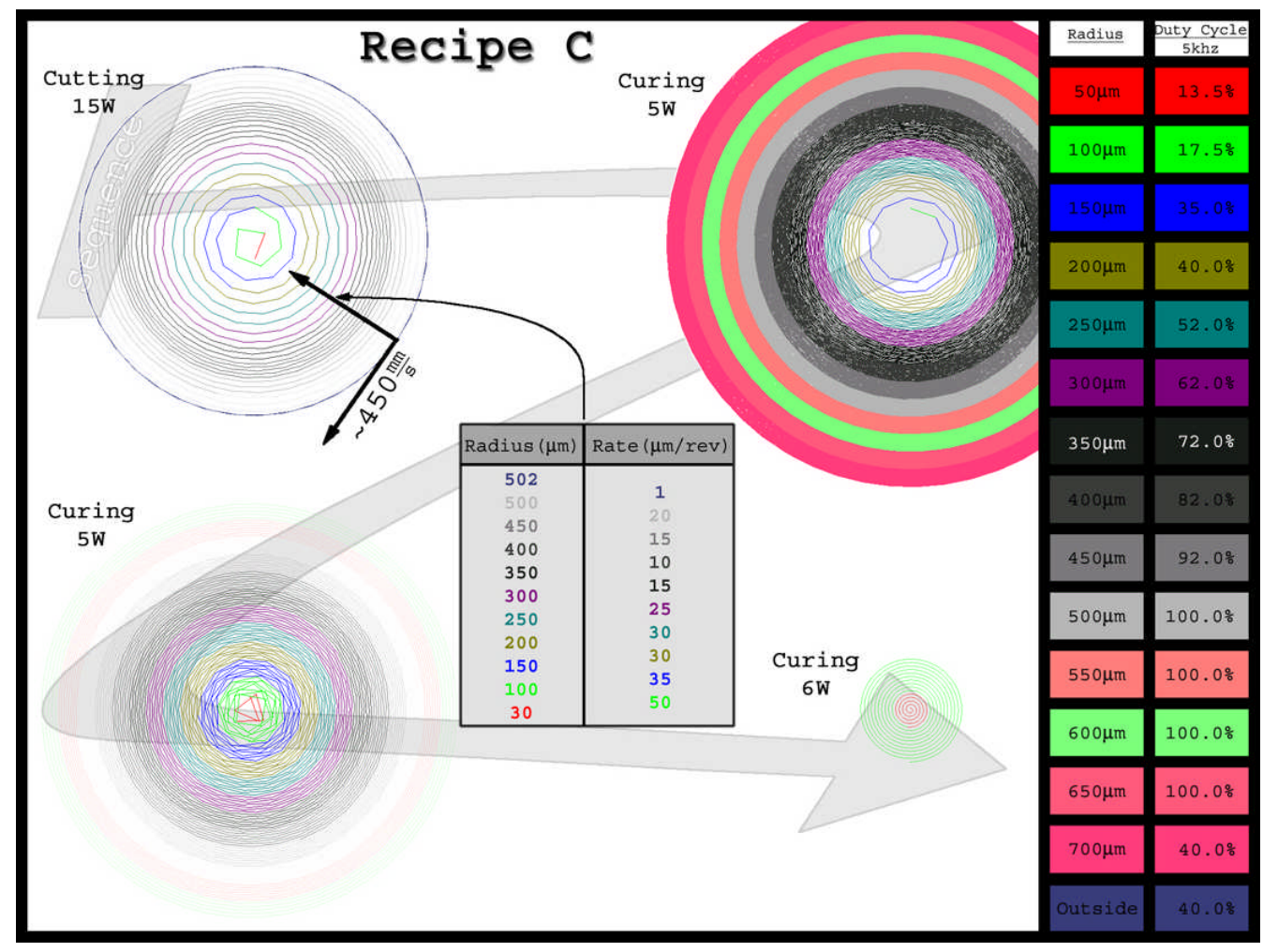

Fig. 5 A schematic representation of a cutting spiral followed by three curing spirals. The cutting spiral goes inward while the three curing spirals all go outward. The pulse repetition frequency was $5 \mathrm{kHz}$ so that a $100 \%$ duty factor corresponds to $\mathrm{CW}$ while a $10 \%$ duty factor corresponds to a $20 \mu$ s pulse length. The duration of the cutting spiral was $\sim 110 \mathrm{~ms}$ during which $\sim 1.3 \mathrm{~J}$ of energy reached the surface.
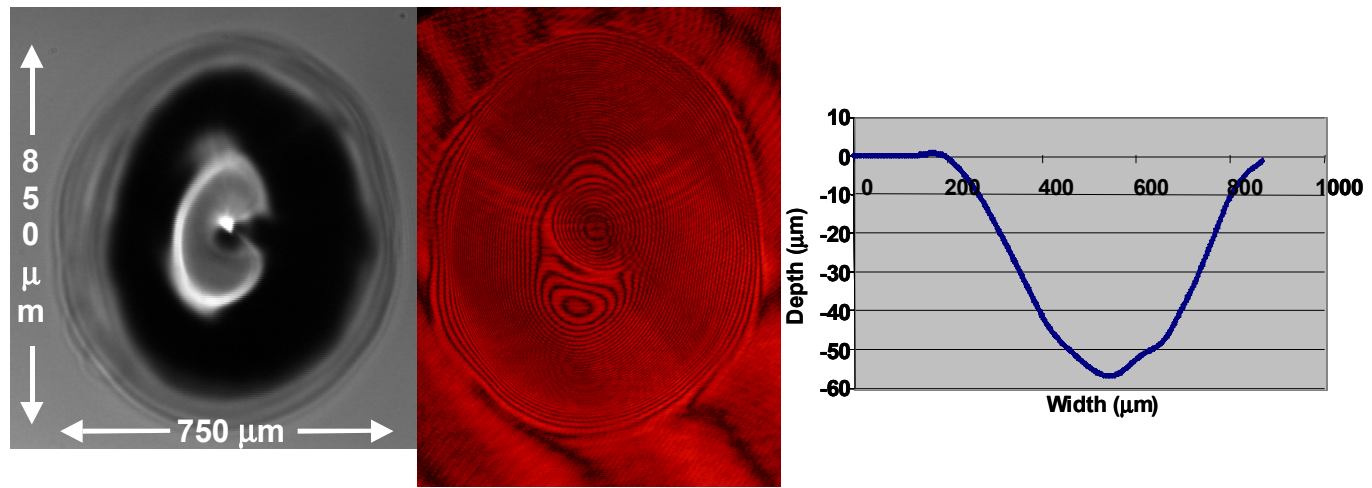

Fig. 6 A microscope image, shearing interferogram, and stylus depth profile of a mitigation site produced by a protocol similar to that of Fig. 5. There are 46 fringes from the perimeter to the bottom giving a depth of $63 \mu \mathrm{m}$ at $1.37 \mu \mathrm{m}$ per fringe, consistent with the $56-\mu \mathrm{m}$ depth measured by stylus profilometry. 


\section{MITIGATION and DAMAGE TESTING OF LASER DAMAGE SITES}

Laser damage sites were initiated on a fused silica sample by raster scanning the $7.5 \mathrm{~ns}, 355 \mathrm{~nm}$ third harmonic beam of a Quanta-Ray, Pro 350GCR, Nd:YAG laser, that was focused onto the exit surface of the sample to a fluence of $\sim 22 \mathrm{~J} / \mathrm{cm}^{2}$. These sites were then grown individually by repeated scans to sizes ranging from $\sim 100 \mu \mathrm{m}$ to $>500 \mu \mathrm{m}$ in transverse dimensions. There were 23 sites in all. Twenty-one are shown in Fig. 7.

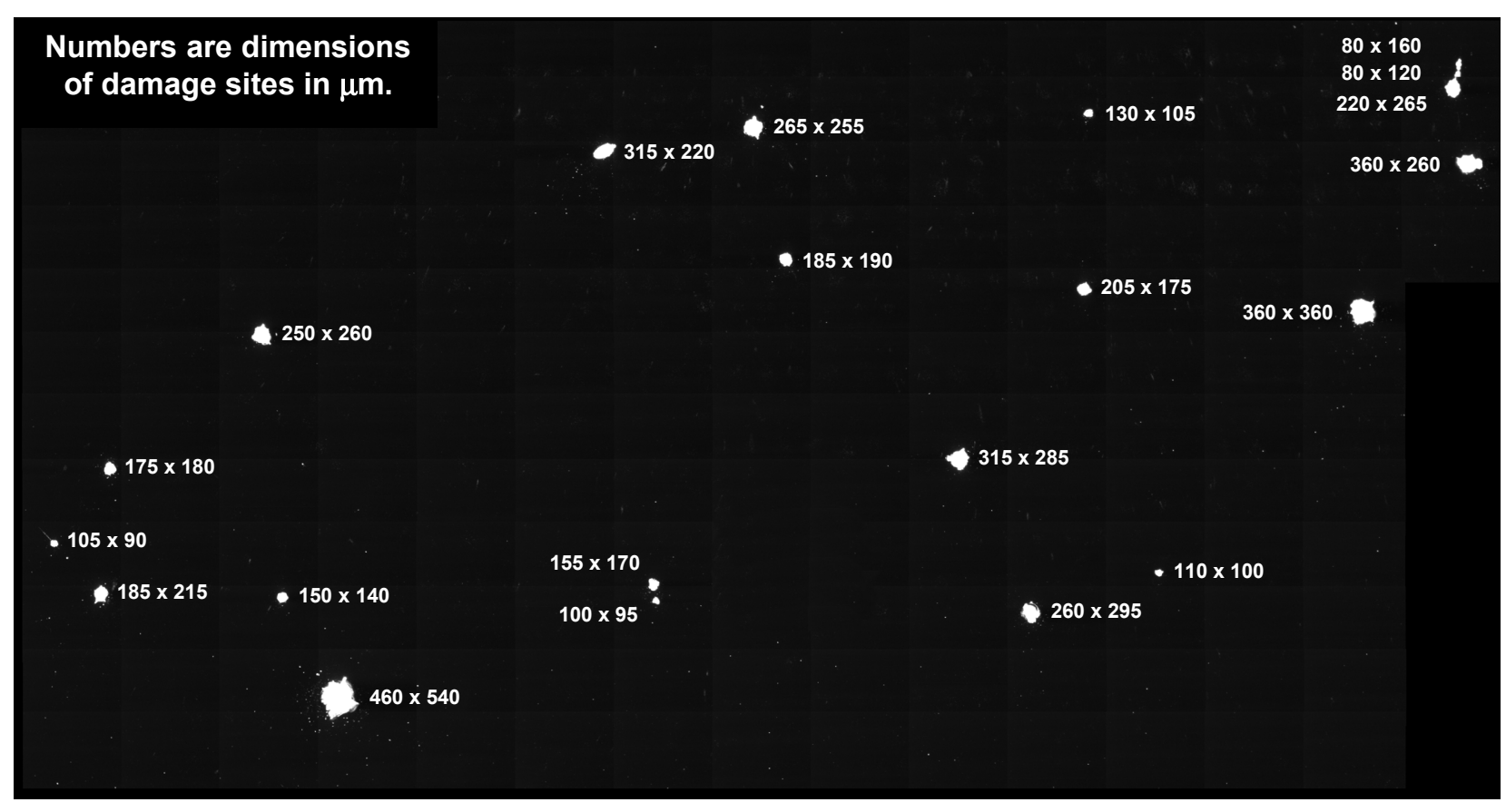

Fig. 7 Twenty-one of the twenty-three damage sites before mitigation.

The damage sites were then mitigated using a spiral cutting and curing protocol similar to that described in section 4. The protocol was repeated until as much indication of the original damage was eliminated from the microscope image as possible. In three cases it was not possible to completely eliminate evidence of the original damage site. Two of these three were the two largest damage sites. Only 20 mitigations were necessary in all because a single mitigation was sufficient in two instances where two and three damage sites were closely grouped respectively.

The mitigated damage sites were then damage tested by raster-scanning the $7.5 \mathrm{~ns}, 355 \mathrm{~nm}$ beam of the Quanta-Ray Nd:YAG laser over them at successively higher fluences. Fig. 8 shows that 17 of the 20 mitigated sites survived without damage to a fluence of $22 \mathrm{~J} / \mathrm{cm}^{2}$ (equivalent to the highest fluence expected in the NIF UV beam at $3 \mathrm{~ns}$ ). The three sites that damaged were the same three where the original damage was not completely removed by mitigation. The largest one of these is shown in Fig. 9 before and after mitigation, and after damage testing. Only one of the three was less than $300 \mu \mathrm{m}$ in size. The largest damage site that was successfully mitigated is shown in Fig. 10 before and after mitigation, and after damage testing. It can be seen that the damage was completely removed by mitigation. 


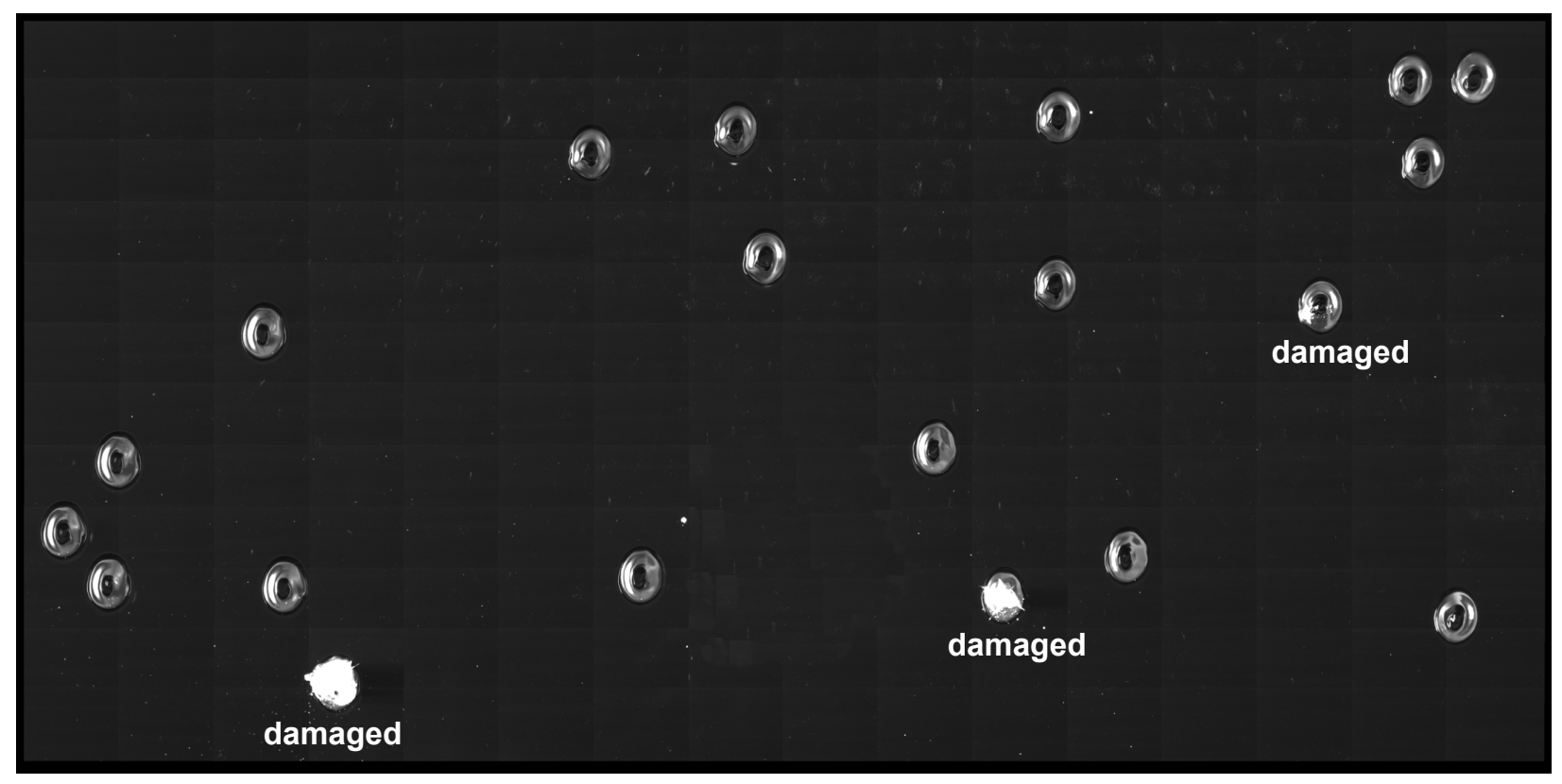

Fig. 8 Three of the twenty mitigated damage sites damaged after raster scanning the 7.5 $\mathrm{ns}, 355 \mathrm{~nm}$ beam over them at a fluence of $22 \mathrm{~J} / \mathrm{cm}^{2}$. These were the only three sites where evidence of the original damage remained in the microscope image after mitigation.
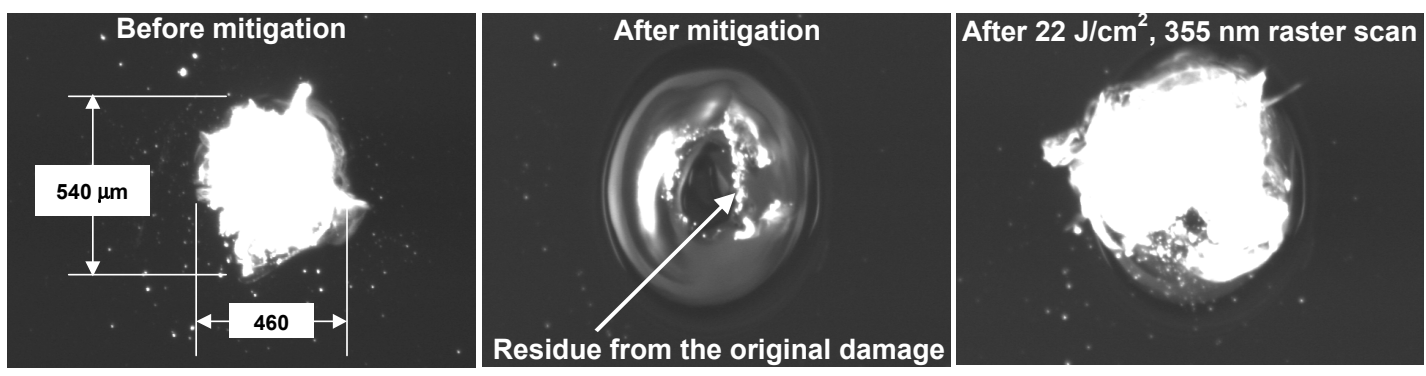

Fig. 9 The largest mitigated site failed when damage tested at $355 \mathrm{~nm}$ as seen in the microscope images before and after mitigation, and after damage testing. The original damage is clearly evident after mitigation. This is also true of the other two sites that damaged after mitigation.
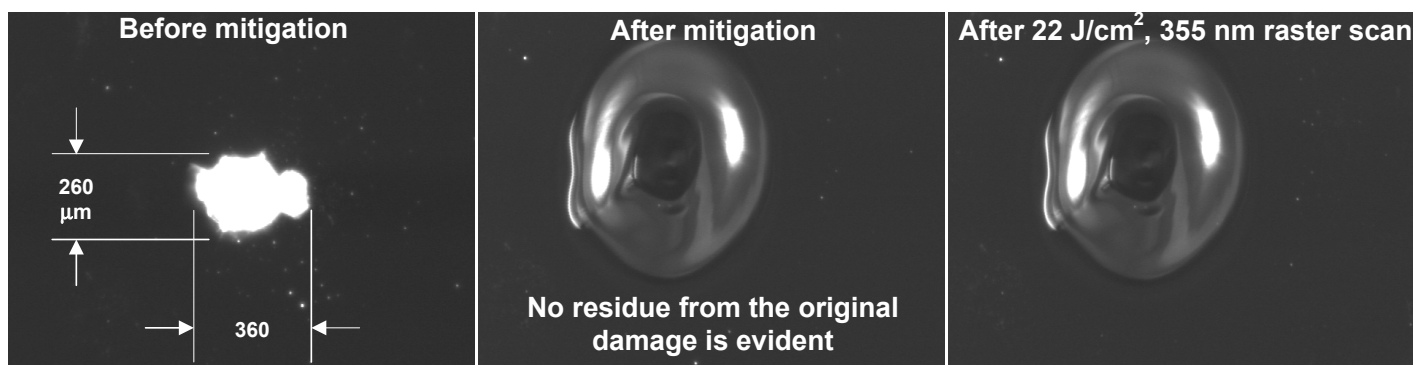

Fig. 10 The largest mitigated site that survived damage testing at $355 \mathrm{~nm}$ as seen in the microscope images before and after mitigation, and after damage testing. Evidence of the original damage is completely removed by mitigation. This is also true of the other 16 sites that survived after mitigation. 


\section{SUMMARY}

Economic operation of the NIF at LLNL is strongly dependent on the ability to mitigate growth of laser surface damage to the high-cost final optics by the $351 \mathrm{~nm}$, third harmonic beam. Melting and evaporation by one or more shots of a focused, $10.6 \mu \mathrm{m} \mathrm{CO}_{2}$ laser beam has previously been shown to successfully mitigate growth provided the damage site is $<100 \mu \mathrm{m}$ in size. However, this method fails for larger sites because cracks would propagate outward from the damage during mitigation or damage testing at $355 \mathrm{~nm}$.

A new technique was developed and demonstrated to successfully mitigate damage growth of sites as large as $300 \mu \mathrm{m}$. It utilized a fast, 2-axis, galvanometer beam scanner to scan the $\mathrm{CO}_{2}$ spot in an inward moving spiral pattern. By starting the spiral outside the perimeter of the damage, crack propagation was inhibited. A vacuum nozzle was used to minimize re-deposited or re-condensed fused silica debris that could lead to laser damage. The debris was also re-melted, or cured using lower power spiral scans.

This mitigation and curing protocol was tested on 20 laser damage sites ranging in size from $\sim 100 \mu \mathrm{m}$ to $>500 \mu \mathrm{m}$. Seventeen of the twenty did not damage during subsequent damage testing at $355 \mathrm{~nm}$ with 22 $\mathrm{J} / \mathrm{cm}^{2}, 7.5 \mathrm{~ns}$ pulses (equivalent to the highest fluence expected in the $351 \mathrm{~nm}$ NIF beam). The largest of these was $360 \mu \mathrm{m}$. The 3 sites that damaged were all $>260 \mu \mathrm{m}$ in size. For each of these, residue from the original damage could still be seen in the microscope image after mitigation, while the damage was completely removed by the mitigation process for all the sites that did not damage.

Work will continue to improve and develop new scanning protocols suitable for mitigation of larger sites and more precise control of the shape of the mitigation site.

\section{REFERENCES}

1. L.W.Hrubesh, M.A.Norton, W.A.Molander, E.E.Donohue, S.M.Maricle, B.M.Penetrante, R.M.Brusasco, W.Grundler, J.A.Butler, J.W.Carr, R.M.Hill, L.J.Summers, M.D.Feit, A.Rubenchik, M.H.Key, P.J.Wegner, A.K.Burnham, L.A. Hackel and M.R.Kozlowski, "Methods for mitigating surface damage growth on NIF final optics", Laser-InducedDamage in Optical Materials: 2001, Proc. SPIE 4679, (2002).

2. R. M. Brusasco, B. M. Penetrante, J. A. Butler and L. W. Hrubesh, "Localized CO2 laser treatment for mitigation of $351 \mathrm{~nm}$ damage growth on fused silica", Laser-InducedDamage in Optical Materials: 2001, Proc. SPIE 4679, 40-47 (2002). 